\title{
Surgical results of unifocalization revision
}

Richard D. Mainwaring, MD, ${ }^{\mathrm{a}}$ William L. Patrick, MD, ${ }^{\mathrm{a}}$ Tatiana R. Rosenblatt, BA, ${ }^{\mathrm{a}}$ Michael Ma, MD, Komal Kamra, MD, ${ }^{\mathrm{b}}$ Alisa Arunamata, MD, ${ }^{\mathrm{c}}$ and Frank L. Hanley, $\mathrm{MD}^{\mathrm{a}}$

\section{ABSTRACT}

Objective: Midline unifocalization has been developed for the surgical treatment of pulmonary atresia with ventricular septal defect and major aortopulmonary collateral arteries. All patients will eventually require reoperation due to the presence of a conduit, and some may also require revision of the distal unifocalized bed. The purpose of this study was to evaluate the surgical results of unifocalization revision.

Methods: This was a retrospective review of 254 patients who underwent midline unifocalization for treatment of pulmonary atresia with ventricular septal defect and major aortopulmonary collateral arteries. Forty-eight of 254 patients (18\%) have subsequently undergone unifocalization revision. Thirty-two of these patients had previously undergone a single-stage complete repair, whereas 16 had a unifocalization and placement of a central shunt.

Results: For the 32 patients who previously underwent a complete repair, there have been no early or late deaths. The peak systolic pulmonary artery to aortic pressure ratio was $0.44 \pm 0.11$ after the initial repair and increased to $0.82 \pm 0.18$ before revision. The pressure ratio decreased to $0.41 \pm 0.09$ after revision. Three of 32 patients $(9 \%)$ have subsequently undergone a second unifocalization revision. The 16 patients who previously had a unifocalization/shunt underwent unifocalization revision and complete repair $(n=14)$ and revision and repeat shunt $(\mathrm{n}=2)$. There was 1 operative mortality and 3 late deaths ( $25 \%$ total) in this cohort. Three $(25 \%)$ of 12 survivors have subsequently undergone a second unifocalization revision.

Conclusions: The data demonstrate that unifocalization revision can be performed with a successful outcome in a majority of patients. Patients who under-

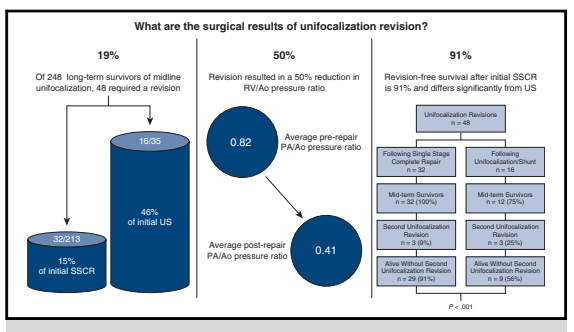

Results for the 48 patients who underwent unifocalization revision.

\section{Central Message}

Unifocalization revision can be performed with a successful outcome in a majority of patients. Patients who underwent an initial single-stage complete repair had the highest success rate.

\section{Perspective}

Midline unifocalization has been developed for the treatment of patients with PA/VSD/MAPCAs. All of these patients will eventually require a reoperation due to the presence of a conduit. However, some patients will also require revision of their unifocalization. The data indicate that the majority of patients achieved a successful outcome following unifocalization revision.

See Commentary on page 546. went an initial unifocalization/shunt had a higher failure rate than patients who were initially repaired. (J Thorac Cardiovasc Surg 2019;158:534-45)

The midline unifocalization approach was developed by Hanley and colleagues ${ }^{1}$ for the surgical treatment of pulmonary atresia with ventricular septal defect and major aortopulmonary collateral arteries (PA/VSD/MAPCAs). This approach is quite versatile and can be utilized for the wide heterogeneity of MAPCA and pulmonary artery

From the Divisions of ${ }^{\mathrm{a}}$ Pediatric Cardiac Surgery, ${ }^{\mathrm{b}}$ Pediatric Cardiac Anesthesia, and ${ }^{\mathrm{c}}$ Pediatric Cardiology, Stanford University School of Medicine, Lucile Packard Children's Hospital at Stanford, Stanford, Calif.

Read at the 98th Annual Meeting of The American Association for Thoracic Surgery, San Diego, California, April 28-May 1, 2018.

Received for publication April 16, 2018; revisions received Sept 7, 2018; accepted for publication Sept 26, 2018; available ahead of print April 11, 2019.

Address for reprints: Richard D. Mainwaring, MD, Division of Pediatric Cardiac Surgery, Stanford University School of Medicine, 300 Pasteur Dr, Falk CVRC, Stanford, CA 94305 (E-mail: mainwaring@stanford.edu).

$0022-5223 / \$ 36.00$

Copyright (c) 2018 by The American Association for Thoracic Surgery https://doi.org/10.1016/j.jtcvs.2018.09.135 anatomy. $^{2-9}$ We recently summarized an experience with 307 patients undergoing primary surgical treatment in which $93 \%$ of patients ultimately achieved complete repair. ${ }^{10}$ The mean right ventricle to aortic peak systolic pressure ratio (RV:Ao) was $0.36 \pm 0.08$. These data demonstrate that a majority of patients with PA/VSD/MAPCAs can achieve complete repair with low RV pressures.

The longer-term fate of unifocalized MAPCAs has been the subject of much speculation. We have previously shown that most patients undergoing complete repair of PA/VSD/

Uf

Scanning this QR code will take you to the article title page to access supplementary information. To view the AATS Annual Meeting Webcast, see the URL next to the webcast thumbnail.

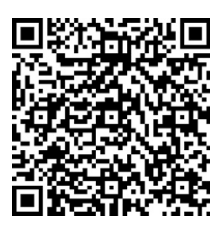




$$
\begin{aligned}
& \text { Abbreviations and Acronyms } \\
& \text { Ao = aorta } \\
& \text { MAPCAs = major aortopulmonary collateral } \\
& \text { arteries } \\
& \text { PA }=\text { pulmonary atresia } \\
& \mathrm{RV} \quad=\text { right ventricle } \\
& \text { VSD }=\text { ventricular septal defect }
\end{aligned}
$$

MAPCAs have little change in RV:Ao over time, ${ }^{11}$ as documented by a comparison of these values in the same patient at initial repair $(0.39 \pm 0.07)$ and after conduit replacement $(0.36 \pm 0.07)$. This cohort of 80 patients was a select subgroup of patients because they had achieved complete repair status and had also survived the complete repair procedure, the interim period, and the conduit change. The inference from these data was that the reconstructed MAPCA bed maintained growth commensurate with the somatic growth in a majority of patients.

All patients who undergo complete repair of PA/VSD/ MAPCAs will eventually require a reoperation due to the presence of an RV to pulmonary artery conduit. ${ }^{12}$ These patients demonstrate a gradual rise in RV pressures over time, and when the pressure ratios reach approximately two-thirds systemic, we recommend performing a cardiac catheterization to assess the distal unifocalized pulmonary artery and MAPCA anatomy. An overwhelming majority of patients are found to have low pulmonary artery pressures and can undergo routine conduit replacement. However, there is a smaller subset of patients who will resurface with evidence of elevated pulmonary artery pressures. In this circumstance, cardiac catheterization is used to define abnormalities in the unifocalized pulmonary artery and MAPCA bed. It has been our view that surgical reconstruction would be the optimal treatment of significant distal stenoses, with the caveat that there have been no data to either confirm or refute the validity of this approach. We recently evaluated the factors associated with the need for unifocalization revision. ${ }^{13}$ Having answered that question, the next logical question is how well unifocalization revision actually works. The purpose of this study was to evaluate the surgical results achieved with the unifocalization revision procedure.

\section{MATERIALS AND METHODS}

This was a retrospective review of patients who had the diagnosis of PA/ VSD/MAPCAs and underwent a primary midline unifocalization at Stanford. The study was approved by the Institutional Review Board at Stanford University (protocol ID 33924). There were 254 patients (123 men and 131 women) who met these criteria during the period of time from 2001 through September 2017. Eighty-eight patients (34\%) had DiGeorge syndrome (22q11 deletion), and 7 patients (3\%) had Alagille syndrome. The median age at the time of midline unifocalization was 4.5 months (range, 2 weeks11 years).

Excluded from this analysis are patients who underwent their primary surgical procedure at Stanford during this same time frame but were assigned to a procedure other than midline unifocalization. This includes patients who had dual supply MAPCAs and underwent an initial aortopulmonary window or other palliative procedures. A majority of patients who had an initial aortopulmonary window never required a subsequent unifocalization of MAPCAs. Collectively, patients undergoing procedures other than a midline unifocalization represent about $20 \%$ of the entire MAPCA surgical experience at Stanford, and this accounts for the numerical difference between the 307 patients included in our previous article $^{10}$ and the current review. We also excluded all patients who underwent a surgical procedure at an outside institution before referral to Stanford. These patients typically have undergone procedures that we would not perform at Stanford, have lost multiple segments of lung, and are referred due to a failure to progress at their home institution. Under these circumstances, the procedures are largely rehabilitative in nature, do not include unifocalization of MAPCAs, but instead require revisions of proximal and distal abnormalities. As a consequence, the prognosis for these rehabilitative efforts is somewhat poorer than when a full complement of lung segments is available.

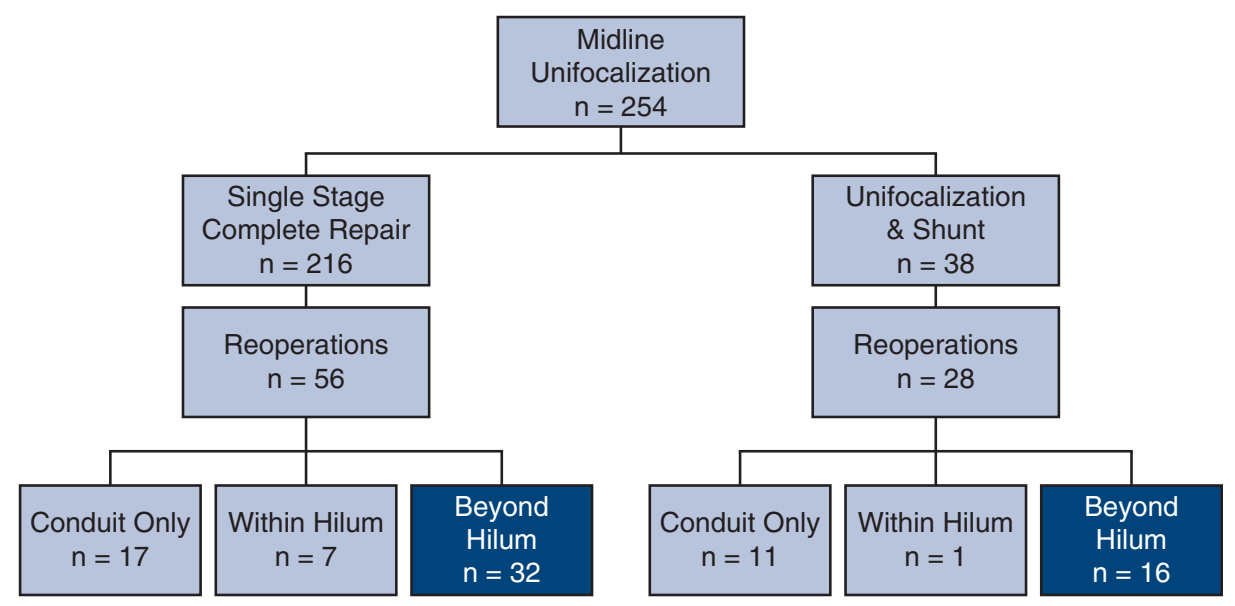

FIGURE 1. Flow diagram for the 254 patients who underwent a midline unifocalization. Two hundred sixteen patients ( $85 \%)$ had a single stage complete repair and 38 patients $(15 \%)$ had a unifocalization shunt. Eighty-four patients have subsequently undergone reoperations at Stanford, of whom 48 required unifocalization revisions beyond the hilum (blue boxes). 
The patients had a mean of $4.0 \pm 1.2$ MAPCAs. This included $2.2 \pm 1.0$ MAPCAs to the right lung (range, 1-5) and $1.8 \pm 0.8$ MAPCAs to the left lung (range, 1-4). Sixty-eight patients had absent central pulmonary arteries, including 46 with predominantly single-supply MAPCAs and 22 with either a ductus arteriosus or hemitruncus to 1 lung and MAPCAs to the contralateral lung.

Two hundred sixteen of 254 patients $(85 \%)$ underwent a single-stage complete repair, including complete closure of the VSD and placement of an aortic valved conduit from the RV to reconstructed pulmonary bed. A transthoracic pressure line was placed in all patients who underwent a complete repair, and RV:Ao recorded at the conclusion of that operation. The mean RV:Ao for these 216 patients was $0.36 \pm 0.09$. There were 3 operative deaths and 8 late deaths in this cohort. Thirty-eight patients $(15 \%)$ underwent unifocalization and placement of a central shunt from the ascending aorta to reconstructed pulmonary bed. There were 3 operative deaths and 7 late deaths in this cohort. The mean RV:Ao for 24 patients who subsequently underwent complete repair was $0.42 \pm 0.07$. The survivors have been followed for a mean of $4.5 \pm 1.2$ years.

Eighty-four patients have subsequently undergone a reoperation at Stanford University. This includes 56 patients who underwent a single-stage complete repair and 28 patients who underwent a unifocalization and shunt (Figure 1). For 56 patients who had a previous complete repair, the subsequent operations were conduit changes only in 17 patients, conduit changes and revisions within the hilum in 7 patients, and conduit changes and revisions beyond the hilum of the unifocalized bed in 32 patients. For 28 patients who underwent a previous unifocalization and shunt, the subsequent operations were complete repair with conduit placement in 11 patients, complete repair with conduit placement in conjunction with surgical revision confined to within the hilum in 1 patient, and revision of the distal unifocalized bed beyond the hilum with or without concomitant complete repair in 16 patients. The median interval between initial unifocalization and revision was 12 months, compared with 31 months for the 35 patients who underwent reoperations but did not require unifocalization revision.

The 48 patients who required revisions of the unifocalized bed beyond the hilum are the focus of the remainder of this study (Table 1). Thirty-two patients had undergone a previous complete repair and returned with a mean pulmonary artery to Ao pressure ratio of $0.82 \pm 0.18$ (range, 0.36-1.41) at catheterization. This was an increase of $0.38 \pm 0.08$ (range, 0.02-0.87) compared with the values measured in the operating room at the time of initial repair (Figure 2). There were 14 patients who had pulmonary artery to Ao pressure ratio above 0.75 and were categorized as "severe." Ten patients had pulmonary artery to Ao pressure ratio between 0.50 and 0.75 and were categorized as "moderate." All 24 moderate and severe patients were known to have elevated RV pressures based on noninvasive studies, representing at a median of 9 months (range, 1-17 months) following complete repair. There were 8 patients who had pulmonary artery to Ao pressure ratio $<0.50$, and thus were categorized as "mild." Three of these 8 patients had an abnormal lung perfusion scan, whereas 5 patients were not perceived to have a problem with the unifocalized bed before their cardiac catheterization. These 8 patients represented at a median of 27 months (range, 7-54 months) following complete repair.

There was a significant correlation $(R=0.73 ; P<.05)$ between pulmonary artery to Ao pressure ratio and the number of obstructed lung segments defined at catheterization for the 32 single-stage complete repair patients (Figure 3, A). Patients who were characterized as mild had a median of 6 lung segments affected (range, 4-12 segments; interquartile range [IQR], 5-9 segments), moderate had a median of 9 affected segments (range, 6-14 segments; IQR, 8-10 segments), and severe had a median of 13 affected segments (range, 8-18 segments; IQR, 11-14 segments). Overall, the median number of obstructed lung segments was 9 (range, 4-18 segments, IQR, 8-14 segments).

For 16 patients who had undergone a previous unifocalization/shunt, the $\mathrm{RV}$ pressures by definition remain at systemic levels due to presence of an
TABLE 1. Characteristics of the 248 operative survivors following midline unifocalization

\begin{tabular}{|c|c|c|c|}
\hline Characteristic & $\begin{array}{c}\text { Revision } \\
\text { beyond } \\
\text { hilum } \\
(n=48)\end{array}$ & $\begin{array}{c}\text { No revision } \\
\text { beyond } \\
\text { hilum } \\
(n=200)\end{array}$ & $\begin{array}{c}P \\
\text { value }\end{array}$ \\
\hline Single-stage complete repair & 32 & 181 & .001 \\
\hline Unifocalization and shunt & 16 & 19 & \\
\hline RV:Ao after complete repair & $0.44 \pm 0.08$ & $0.33 \pm 0.07$ & .001 \\
\hline Absent central pulmonary arteries & 22 & 47 & .001 \\
\hline Present central pulmonary arteries & 26 & 153 & \\
\hline Male & 25 & 94 & .41 \\
\hline Female & 23 & 106 & \\
\hline \multicolumn{4}{|l|}{ Age } \\
\hline $0-60 \mathrm{~d}$ & 8 & 25 & .14 \\
\hline 2-12 mo & 37 & 138 & \\
\hline$>1 \mathrm{y}$ & 3 & 37 & \\
\hline DiGeorge syndrome & 14 & 72 & .49 \\
\hline No DiGeorge & 34 & 128 & \\
\hline Alagille syndrome & 4 & 3 & .10 \\
\hline No Alagille syndrome & 44 & 197 & \\
\hline Bilateral MAPCAs & 41 & 184 & .14 \\
\hline Ductus or hemitruncus & 7 & 16 & \\
\hline \multicolumn{4}{|l|}{ No. of MAPCAs } \\
\hline 1 or 2 & 6 & 26 & .99 \\
\hline 3 or 4 & 25 & 102 & \\
\hline 5 or 6 & 15 & 62 & \\
\hline 7,8 , or 9 & 2 & 10 & \\
\hline
\end{tabular}

Values are presented as $\mathrm{n}$ or mean \pm standard deviation. $R V: A o$, Right ventricle to aortic peak systolic pressure ratio; MAPCAs, major aortopulmonary collateral arteries.

open VSD. For these patients, it was more difficult to ascertain whether there was a problem with the unifocalized bed based on noninvasive studies. However, some patients may demonstrate an abnormality based on decreasing oxygen saturations or an abnormal lung perfusion scan. The majority of patients in this cohort were undergoing repeat cardiac catheterization to assess the anatomy and physiology to evaluate their candidacy for complete repair (Figure E1, $A-D$ ). Based on the number of lung segments with obstruction demonstrated at catheterization, 4 would be categorized as mild, 8 as moderate, and 4 as severe (Figure 3, B). The median interval between unifocalization/shunt and unifocalization revision was 7 months.

The surgical technique that we have developed for unifocalization revision is performed through a midline sternotomy. The conduit and unifocalized pulmonary artery bed are dissected as much as feasible before institution of bypass. This dissection is performed circumferentially down to the level of the ostia of the basilar segments, taking care to push the lung tissue away from the segmental origins. A typical case is shown in Figure 4, $A$, demonstrating a long-segment stenoses to both the right and left lower lobes and conduit dysfunction. After instituting bypass, the patient is cooled to $25^{\circ} \mathrm{C}$. The conduit is removed and the right and left branch pulmonary arteries are divided (Figure 4, B). The lobar and segmental branches are controlled with vascular clips. The individual 


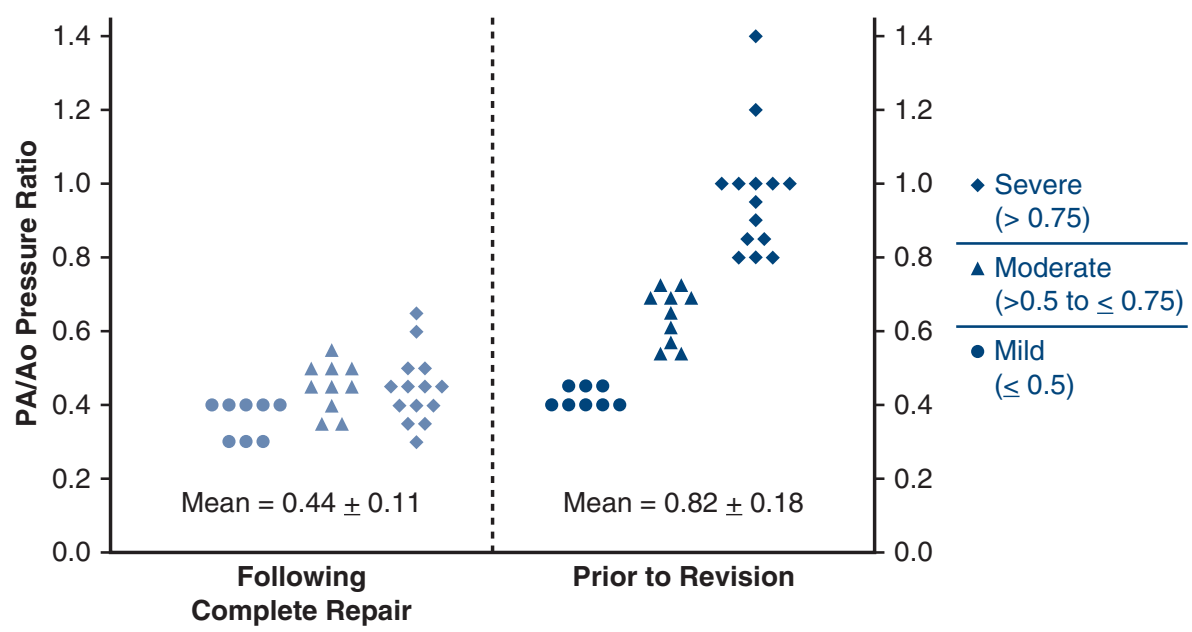

FIGURE 2. Graph displaying the pulmonary artery $(P A)$ to aortic $(A o)$ pressure ratios for the 32 patients who underwent a single stage complete repair and subsequently required unifocalization revision. The first data set shows the PA to Ao pressure ratios immediately following complete repair. The second data shows the PA to Ao pressure ratios at catheterization before unifocalization revision. The legend on the right classifies cases by PA to Ao pressure ratio into mild $(<0.50[n=8])$, moderate $(>0.50-<0.75[n=10])$, and severe $(>0.75[n=14])$.

branch pulmonary arteries are then incised along the inferior and medial border to the lower lobe segmental vessels (Figure 4,C). The lobar and segmental branch pulmonary arteries are individually probed to assess the degree of patency. Although the cardiac catheterization findings most often are confirmed intraoperatively, it should not be assumed that all abnormalities have been delineated, and a thorough assessment may reveal discrepancies that need to be addressed during the surgical procedure. ${ }^{14}$ Pulmonary artery homograft is then used to patch augment the pulmonary artery (Figure $4, D$ ). The branch pulmonary arteries are subsequently brought together and a new Ao homograft placed from the RV to the reconstructed pulmonary arteries (Figure $4, E$ ). These procedures are performed on a beating heart with full flow for $25^{\circ} \mathrm{C}$ (ie, $100 \mathrm{~mL} / \mathrm{kg} / \mathrm{min}$ ) with the exception of the time required for concomitant VSD repair, in which circumstance the Ao is crossclamped and the heart arrested during this phase of the operation.

Results are reported as means \pm standard error, and medians and ranges where appropriate. Statistical analysis to compare groups was performed using Pearson $\chi^{2}$ or Fisher exact test. Pearson product-momentum correlation was used to evaluate the relationship between pulmonary artery to Ao pressure ratios and the number of segments involved in the unifocalization revision surgery.

\section{RESULTS}

For 32 patients who had previously undergone a singlestage complete repair, there was no operative mortality. The RV:Ao decreased from a mean of $0.82 \pm 0.18$ to $0.41 \pm 0.08$ postoperatively $(P<.05)$. These data are shown in Figure 5, $A$. These 32 patients have been followed for an average of $40 \pm 10$ months. There has been no late mortality. However, 3 patients $(9 \%)$ have subsequently required a second unifocalization revision.

For 16 patients who underwent an initial unifocalization/shunt, 14 patients had a complete repair. There was no operative mortality in this cohort. These 14 patients had a postoperative RV:Ao of $0.41 \pm 0.08$. Two patients had a unifocalization revision and repeat shunt, with 1 operative and 1 interim mortality. The 14 operative survivors have been followed for an average of $37 \pm 8$ months. There were an additional 2 late deaths, for a combined mortality of $25 \%$ in this cohort $(P<.01$ compared with patients who initially had a single-stage complete repair). Three of 12 midterm survivors $(25 \%)$ have subsequently required a second unifocalization revision $(P=.30$ compared with patients who initially underwent a single-stage complete repair). Combining these measures of outcome, the percentage of patients undergoing a unifocalization revision, remaining alive, and not requiring a second unifocalization revision was $91 \%$ for the singlestage complete repair cohort versus $56 \%$ for the unifocalization/shunt cohort $(P<.01)$. The flowchart for the 48 patients who underwent unifocalization revision is in Figure 5, $B$.

The 4 postoperative deaths occurred at $1,2,3$, and 8 months following unifocalization revision. The cause of death was postoperative multisystem organ failure in 1 patient, chronic respiratory failure in 2 patients (both had sustained a phrenic nerve injury and required diaphragm plication), and 1 patient who died at home of unknown causes. The 4 patients had undergone 9, 13, 14, and 14 segmental revisions, and thus had been classified as moderate in 1 patient and severe in 3 patient.

The 6 patients who required a second unifocalization revision had this procedure performed at 5 days; $6,8,11$, and 12 months; and 6 years following their unifocalization revision. These patients had initially undergone $9,10,10$, 12,14 , and 16 segmental revisions, and thus had been classified as moderate in 2 patients and severe in 4 patients. At the second revision, these patients underwent $3,8,8,10,13$, and 14 segmental re-revisions. The characteristics of the 4 


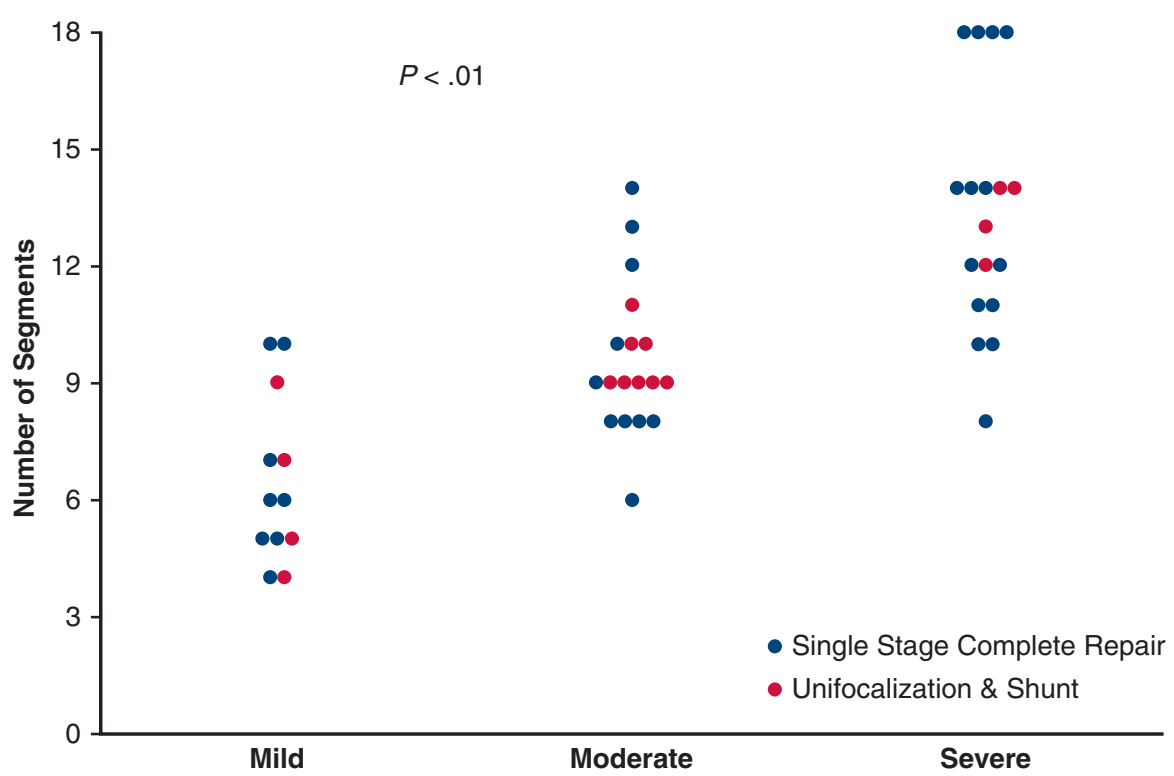

A
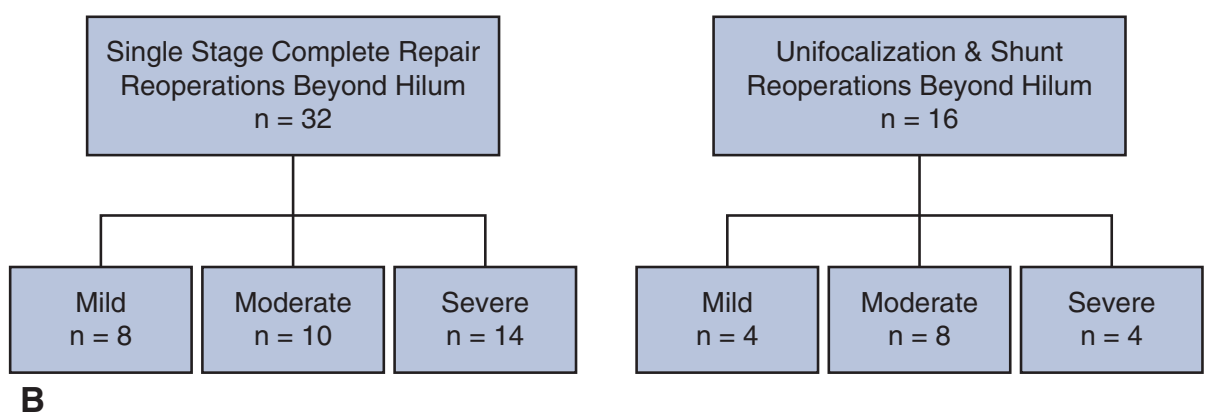

FIGURE 3. A, Graph demonstrating the number of lung segments that were affected by pulmonary arterial stenoses and subsequently underwent unifocalization revision. The data sets are separated into mild, moderate, and severe based on the pulmonary artery to aortic pressure ratios at catheterization before unifocalization revision. The median number of affected lung segments for mild cases was 6 , moderate was 9 , and severe was 13 . The 16 patients who initially had a unifocalization/shunt were categorized according to the number of lung segments affected, because there is no peak systolic pressure data available for these patients. B, Flow chart demonstrating a classification of severity of obstruction for the 32 patients who underwent a single stage complete repair and the 16 patients who had undergone a unifocalization/shunt. For the complete repair patients, 8 were categorized as mild, 10 as moderate, and 14 were severe. For the unrepaired patients, 4 were categorized as mild, 8 as moderate, and 4 as severe.

patients who died and the 6 patients who required a second unifocalization revision are shown in Table 2.

\section{DISCUSSION}

This study was performed to evaluate the surgical outcomes for patients who underwent unifocalization revision following a midline unifocalization. Before revision, the mean pulmonary artery to Ao pressure ratio was $0.82 \pm 0.18$, and the median number of obstructed pulmonary segments was 9 . The data demonstrate that unifocalization revision was associated with an excellent hemodynamic response, as evidenced by a reduction in pulmonary artery to Ao pressure ratios to baseline values. The combined early and late mortality was significantly higher in patients who had undergone an initial unifocalization/ shunt compared with those who had a single-stage complete repair. Overall, $80 \%$ of patients who underwent a unifocalization revision are currently alive and have not required a second unifocalization revision. However, there was a significant disparity in the success rate between patients who initially had a single-stage complete repair $(91 \%)$ and unifocalization/shunt $(56 \%)$. These results are summarized in Figure 6 and Table 1.

We have previously evaluated the risk factors associated with the need for unifocalization revision following midline unifocalization. ${ }^{5}$ That study identified 3 factors, including unifocalization/shunt versus single stage complete repair, higher RV:Ao at completion of repair, and absence of central intrapericardial pulmonary arteries. In addition to identifying the factors that were associated with the need for 

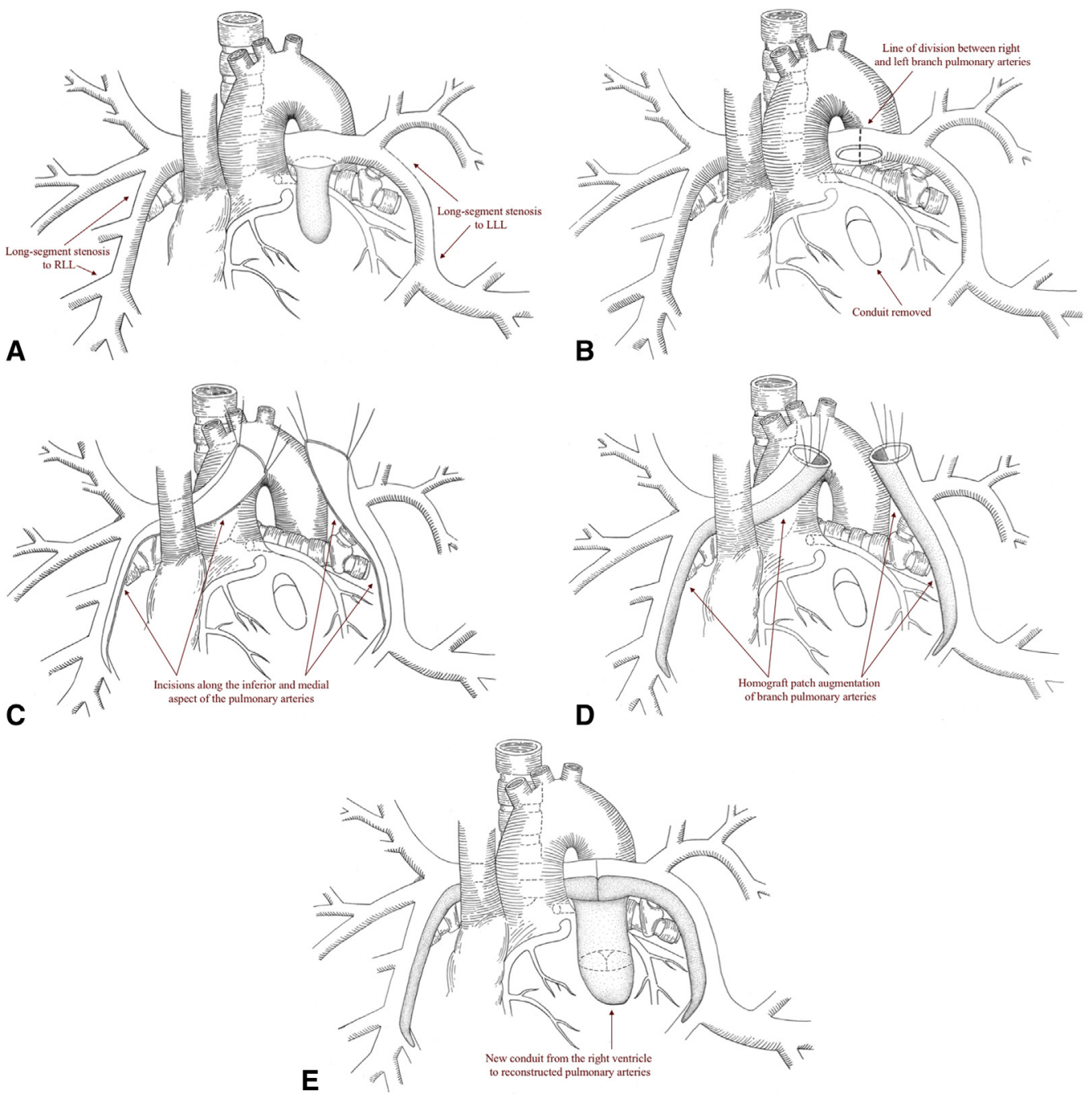

FIGURE 4. A, Illustration demonstrating typical anatomy encountered in patients who require unifocalization revision. There is long-segment stenoses to both the right lower lobe $(R L L)$ and left lower lobe $(L L L)$. In addition, there is conduit dysfunction. B, Illustration demonstrating removal of the conduit and line of division between the right and left pulmonary arteries. C, Illustration demonstrating incisions along the inferior and medial aspect of the branch pulmonary arteries. These incisions are carried beyond the stenoses into the lower lobe medial segmental orifice. D, Illustration demonstrating homograft patch augmentation of the branch pulmonary arteries. E, Illustration demonstrating restoration of continuity between the right and left branch pulmonary arteries. A new aortic valved conduit is placed from the right ventricle to the reconstructed pulmonary arteries. Reproduced with permission from Mainwaring RD, Patrick WL, Ma M, Hanley FL. An analysis of patients requiring unifocalization revision following midline unifocalization for pulmonary atresia with ventricular septal defect and major aortopulmonary collaterals. Eur J Cardiothorac Surg. 2018;54:63-70.

unifocalization revision, that study also demonstrated numerous factors that were not associated with an increased need for revision. Specifically, age, syndrome, unilateral versus bilateral MAPCA anatomy, and number of MAPCAs were not found to be associated with an increased risk of requiring unifocalization revision. Thus, the risk factors for requiring a unifocalization revision have been defined and this risk profile is known at the time of discharge from the hospital.

In the current analysis, 32 of 213 operative survivors $(15 \%)$ who underwent a single-stage complete repair subsequently underwent a unifocalization revision. Onequarter of these patients had mild obstruction, with pulmonary artery to Ao pressure ratios less than half systemic. If one excludes these mild cases from the count, then approximately $11 \%$ of patients who initially underwent a single-stage complete repair required unifocalization revision. For patients who initially underwent a unifocalization/ shunt, $46 \%$ subsequently had a unifocalization revision. The 3 -fold disparity in unifocalization revision between patients undergoing single-stage complete repair versus unifocalization/shunt is not surprising, because the patients who are 


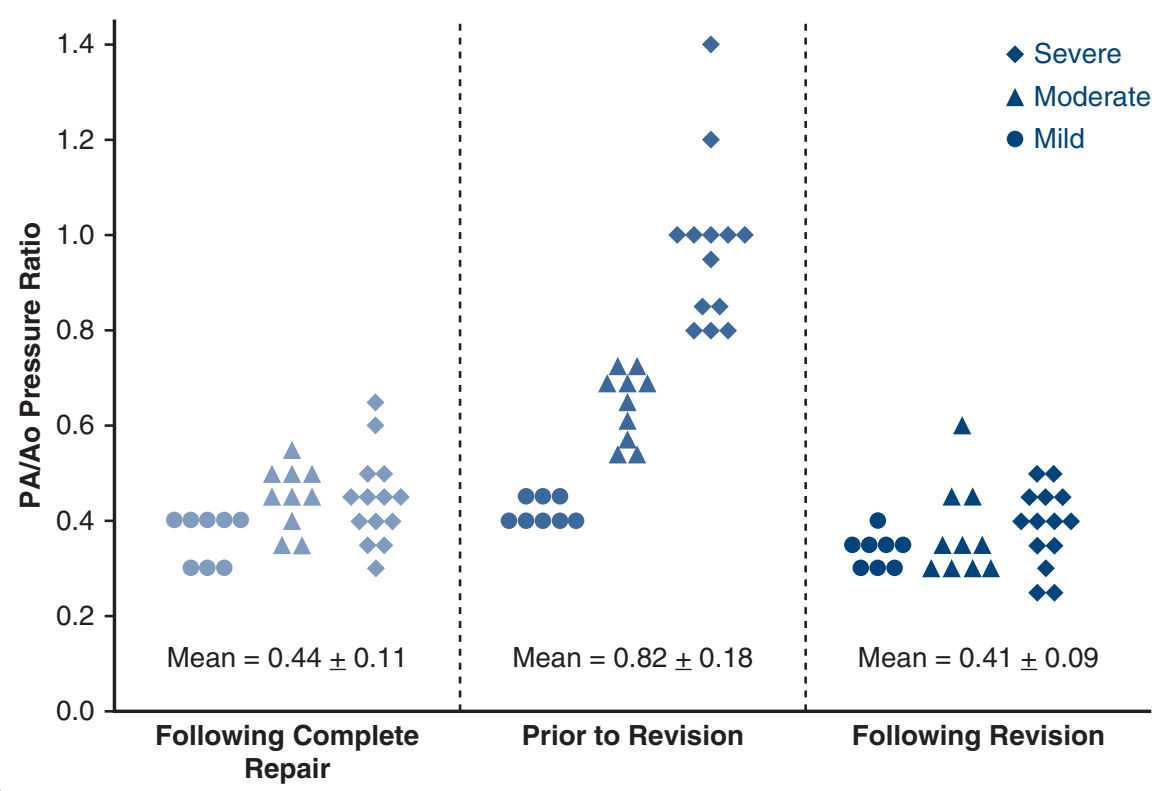

A

Repair

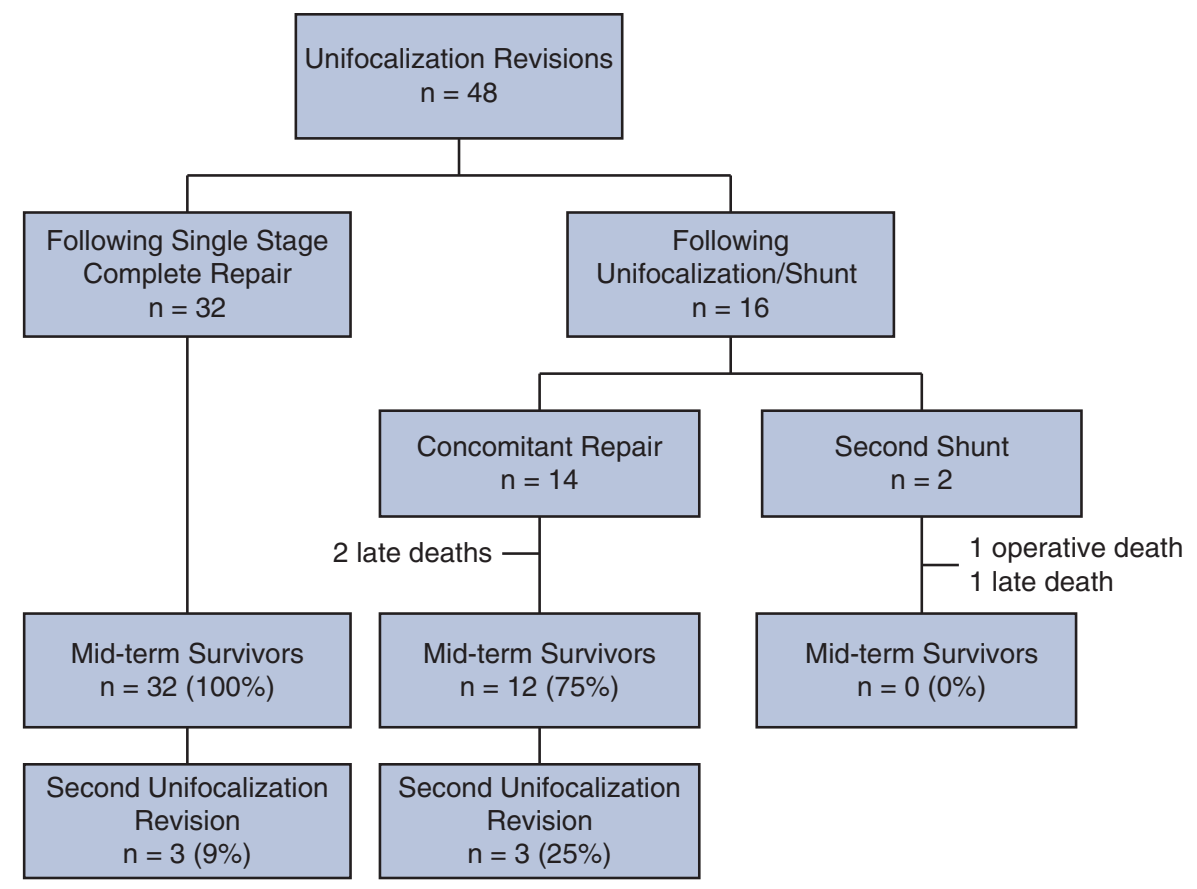

B

FIGURE 5. A, Graph displaying the pulmonary artery $(P A)$ to aortic $(A o)$ pressure ratios for the 32 patients who underwent a single stage complete repair and subsequently required unifocalization revision. Following revision, the PA to Ao pressure ratios decreased to a mean of $0.41 \pm 0.09$. B, Flow chart demonstrating the outcomes for the 48 patients who underwent unifocalization revision. For the 32 patients who initially underwent a single-stage complete repair, there was no early or late mortality. Three patients $(9 \%)$ subsequently required a second unifocalization revision. For 16 patients who initially underwent unifocalization/shunt, there were a total of 4 ( $25 \%)$ early and late deaths $(P<.05$ compared with single-stage complete repair cohort). Three of the 12 survivors $(25 \%)$ subsequently required a second unifocalization revision.

able to undergo a single-stage complete repair have demonstrated 1 or more indicators of a well-developed pulmonary vascular bed. These include higher preoperative oxygen saturations, Qp:Qs > 1.5 at catheterization, or clinical signs of heart failure. For a majority of these patients, we no longer perform an intraoperative flow study because our experience has demonstrated that these indicators are quite reliable. ${ }^{10} \mathrm{In}$ contrast, patients who have lower preoperative oxygen 
TABLE 2. Characteristics of patients who underwent unifocalization revision and either died or required a second unifocalization revision

\begin{tabular}{lcc}
\hline \multicolumn{1}{c}{ Characteristic } & $\begin{array}{c}\text { Deaths } \\
(\mathbf{n = 4})\end{array}$ & $\begin{array}{c}\text { Second } \\
\text { revision } \\
(\mathbf{n}=\mathbf{6})\end{array}$ \\
\hline Single-stage complete repair & 0 & 3 \\
\hline Unifocalization and shunt & 4 & 3 \\
\hline Absent central pulmonary arteries & 2 & 5 \\
\hline Present central pulmonary arteries & 2 & 1 \\
\hline Age at initial surgery & & \\
\hline 0-60 d & 2 & 3 \\
2-12 mo & 2 & 2 \\
\hline DiGeorge syndrome & 0 & 1 \\
\hline No DiGeorge & 3 & 4 \\
\hline Alagille syndrome & 1 & 1 \\
\hline No Alagille syndrome & 0 & 5 \\
Bilateral MAPCAs & 4 & 4 \\
\hline Ductus or hemitruncus & 3 & 2 \\
\hline No. of MAPCAs & 1 & 2 \\
1 or 2 & & 4 \\
3 or 4 & 0 & 0 \\
\hline 7, 6 or 6 & 3 & \\
\hline
\end{tabular}

Values are presented as number of patients. MAPCAs, Major aortopulmonary collateral arteries.

saturations and had a Qp:Qs $<1.0$ at catheterization represent the subgroup of patients for whom we still perform an intraoperative flow study (approximately $20 \%$ of all patients undergoing unifocalization). The intraoperative flow study (pulmonary artery pressure $25 \mathrm{~mm} \mathrm{Hg}$ at a flow of $3.0 \mathrm{~L} /$ $\mathrm{min} / \mathrm{m}^{2}$ ) is designed to identify patients who would have an RV to pulmonary artery peak systolic pressure ratio in excess of 0.50 if the patient underwent complete repair. ${ }^{15}$ In our opinion, these patients are better served with a temporary central shunt, with the possibility that the pulmonary vascular bed may grow and subsequently permit complete repair.

The data demonstrate a correlation between the number of lung segments affected by stenoses and the pulmonary artery to Ao peak systolic pressure ratios (Figure 3, A). This correlation is not surprising because the pulmonary artery pressures will be directly influenced by the number of obstructed segments. It appears that there is an inflection point at 5 or 6 segments, with little change in pulmonary artery pressures below this number. Patients categorized as moderate had a median pulmonary artery pressure ratio of 0.62 and a median of 9 lung segments affected. Patients in the severe category had a median pulmonary artery pressure ratio of 1.0 and a median of 13 lung segments involved. In performing this analysis, we did not attempt to parse the degree of obstruction to the segments, but included all segments that were distal to a pulmonary artery branch that underwent surgical reconstruction. The diagnosis of a hemodynamically significant obstruction at catheterization was defined as a demonstrable pressure drop across an angiographic stenosis. A thorough intraoperative assessment of lobar and segmental branches is conducted by probing the vessels and sometimes reveal abnormalities that were not apparent angiographically. These abnormalities are also addressed while the opportunity is available for surgical reconstruction. Although there is no doubt that the degree of obstruction will influence the pulmonary artery pressure ratios, we believe that the relationship between the number of affected lung segments and pressures is clear without the need for a more complicated classification system.

Our management algorithm for stenoses in a previously unifocalized pulmonary bed is based on a thorough evaluation of the preoperative findings as well as the specific circumstances surrounding each case. Patients with elevated pulmonary artery to Ao pressure ratios will (by correlation) have a considerable number of segments that have a proximal obstruction. These cases have an absolute mandate for intervention, and we would favor a surgical approach in this setting because it is our belief that patch augmentation will be more efficacious in permanently relieving obstruction than would be achieved through interventional catheterization techniques. We also favor surgical treatment of milder forms of obstruction that are discovered during the preoperative workup of patients with conduit dysfunction. In this case, the indication for surgery is the conduit replacement, and the unifocalization revision can effectively be performed as a concomitant procedure. In contrast, there are cases where milder forms of obstruction are discovered during routine diagnostic catheterization, and in the setting of well-functioning conduit, it is reasonable to proceed with balloon dilation of the stenosis. ${ }^{16-18}$

Following discharge from the hospital after midline unifocalization, we have implemented a surveillance program to detect any significant changes in clinical status. This includes echocardiogram and oxygen saturation at 3 months, and these 2 studies plus a lung perfusion scan at 6 months. An echocardiogram and oxygen saturation measurement are repeated at 9 months, and we recommend performing a cardiac catheterization at 1 year following surgery to visualize the reconstructed pulmonary artery bed.

Patients who required unifocalization revision underwent this reoperation at a median of 12 months postoperatively compared with 31 months for patients who underwent reoperations that did not require unifocalization revision. This finding suggests the changes in the pulmonary vascular bed that ultimately necessitate unifocalization revision occur relatively early. During these reoperations, we frequently dissect well beyond the boundaries of the original procedure, particularly when there is lower lobe 


\section{What are the surgical results of unifocalization revision?}

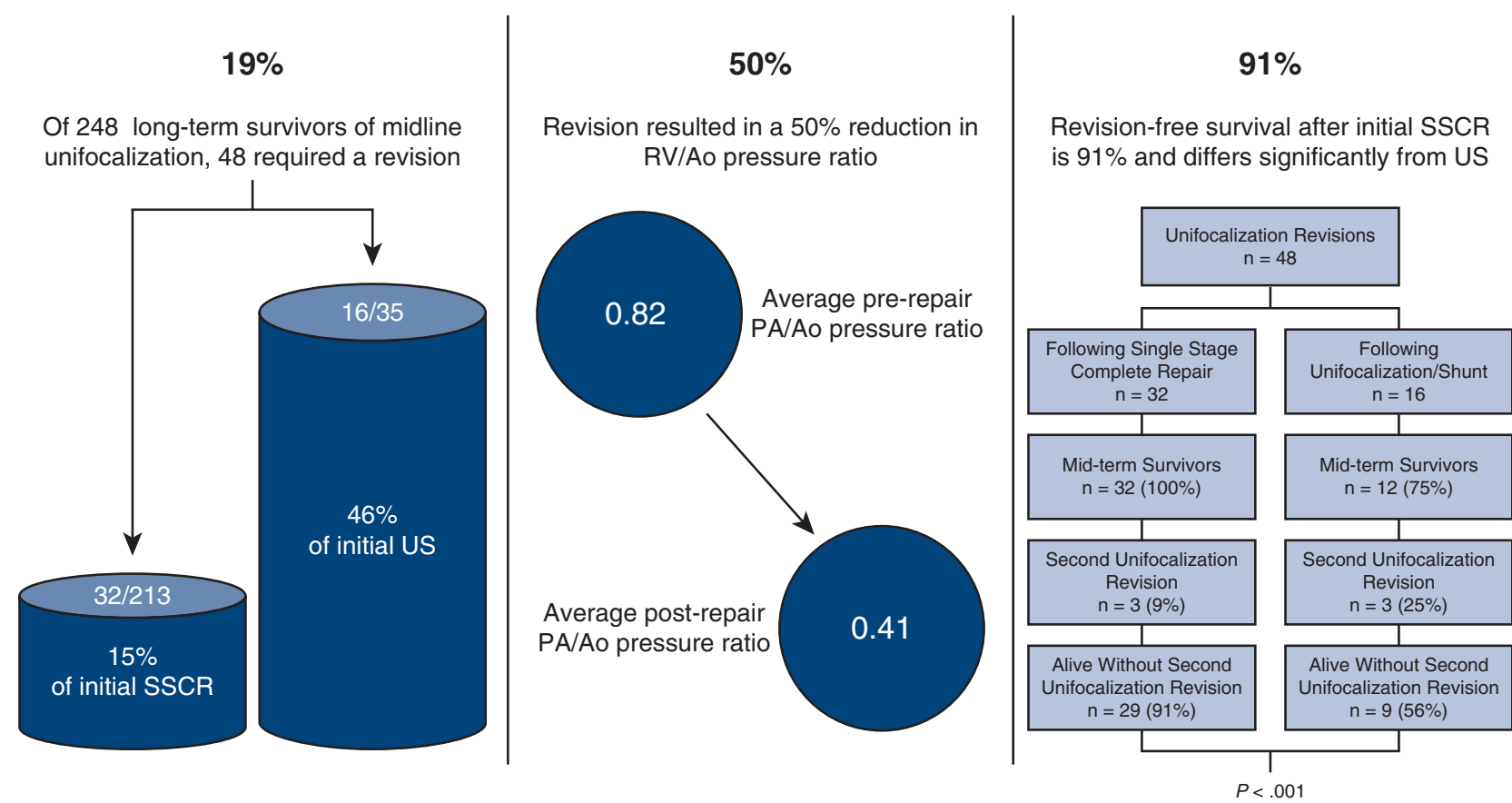

FIGURE 6. Summary of the surgical results of unifocalization revision. For 248 survivors following an initial midline unifocalization, $19 \%$ have subsequently required unifocalization revision. This included $15 \%$ of patients who initially had a single-stage complete repair (SSCR) versus $46 \%$ of patients who initially had a unifocalization and shunt $(U S)$. Unifocalization revision resulted in a $50 \%$ reduction in the pulmonary artery to aortic $(P A / A o)$ pressure ratios. Ninety-one percent of patients who initially had a single-stage complete repair and then underwent unifocalization revision are alive and have not required a second unifocalization revision. In contrast, only $56 \%$ of patients who initially underwent US and then underwent unifocalization revision are alive and have not required a second unifocalization revision $(P<.001$ compared with the single-stage cohort). $R V / A o$, Right ventricle to aortic.

obstruction. It is our observation that we can rarely discern the underlying cause of the problem (ie, surgical vs native tissue stenoses). The exception is when the anatomic issue is beyond the boundaries of the previous unifocalization, in which case it is evident that the source of the problem must be in the native tissue.

There were 6 patients who have subsequently undergone a second unifocalization revision, including 3 patients from the single-stage complete repair cohort and 3 patients from the unifocalization/shunt cohort. These 6 patients all had significant amounts of obstruction repaired at the time of their unifocalization revision. Five of 6 patients re-presented within a year, and these patients underwent surgical repair of $3,8,8,10,13$, and 14 recurrent segmental obstructions. It is noteworthy that the characteristics of these 6 patients (listed in Table 2) do not reveal any specific risk factors. The midterm and long-term fate of patients who have undergone a second unifocalization revision is currently unknown.

Skeptics of the unifocalization approach have pointed out that MAPCAs are neither embryologically nor anatomically normal pulmonary artery vessels. ${ }^{19}$ Authors have raised concerns over the longer-term viability of unifocalized MAPCAs. ${ }^{20-22}$ Although there is no doubt that MAPCAs differ widely from pulmonary arteries, there is also no evidence to suggest that unifocalized MAPCAs will behave any better or worse than pulmonary arteries with abnormal architecture that require extensive surgical reconstruction. ${ }^{23-28}$ There is the additional confounding factor that approximately $20 \%$ of patients with MAPCAs do not have any central pulmonary arteries, ${ }^{2,6,7}$ meaning the MAPCAs are the sole source of pulmonary blood flow regardless of any theoretical preferences or concerns. For this subset of patients, unifocalization of MAPCAs is the only option to achieve a pulmonary vascular bed adequate for complete repair.

Although our preference is to surgically manage most stenoses of a unifocalized pulmonary vascular bed, there is also an important and complimentary role for interventional catheterization techniques. Discrete peripheral stenosis may be amenable to balloon dilation, particularly in the setting of low pulmonary artery pressures and a conduit that is functioning satisfactorily. In this situation, there is no immediate indication for surgical intervention, and thus balloon dilation is a reasonable choice. Stenoses in the central branch pulmonary arteries may also respond well to balloon dilation, and potentially may result in a reduction of RV pressures and/or relief of maldistribution of pulmonary blood flow. We strongly discourage the use of 
pulmonary stents because they invariably develop neointimal fibrosis and create a situation that is far more difficult to treat than without a stent. Interventional catheterization techniques may be particularly useful in treating conduit stenosis, which is the most common cause of failure in young patients repaired during infancy due to the relatively small size of the conduits that can be placed at that age. Conduit stenosis may be amenable to balloon dilation or stenting to increase the longevity of the conduit. Finally, in recent years we have increasingly utilized the transcatheter Melody valve (Medtronic, Minneapolis, Minn) as an option for pulmonary valve replacement in children with conduits of an appropriate size.

\section{CONCLUSIONS}

Our article summarizes the results for patients undergoing unifocalization revision following a midline unifocalization. The data demonstrate that approximately four fifths of patients had a lasting benefit from this surgical procedure. It is also evident that patients who underwent an initial unifocalization/shunt have a less-favorable outcome compared with patients who underwent a single-stage complete repair. The risk factors for favorable and unfavorable surgical outcomes for PA/VSD/MAPCA are steadily being elucidated and should provide important information to guide treatment plans in the future.

\section{Webcast}

You can watch a Webcast of this AATS meeting presentation by going to: https://aats.blob.core.windows.net/ media/18AM\%20Re-edits/S52_4_webcast_040806475_ Reedit.mp4.

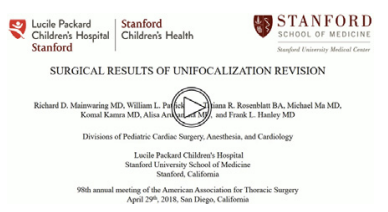

\section{Conflict of Interest Statement}

Authors have nothing to disclose with regard to commercial support.

The illustrations demonstrating the surgical technique for unifocalization revision (Figure 3, A-E) were drawn by Erin Anne Mainwaring.

\section{References}

1. Reddy VM, Liddicoat JR, Hanley FL. Midline one-stage unifocalization and repair of pulmonary atresia with ventricular septal defect and major aortopulmonary collaterals. J Thorac Cardiovasc Surg. 1995;109:832-45.

2. Carrillo SA, Mainwaring RD, Patrick WL, Bauser-Heaton H, Peng L, Reddy VM, et al. Surgical repair of pulmonary atresia/ventricular septal defect/major aortopulmonary collaterals with absent intra-pericardial pulmonary arteries. Ann Thorac Surg. 2015;100:606-14.
3. Mainwaring RD, Reddy VM, Hanley FL. Surgical reconstruction of pulmonary stenosis with ventricular septal defect and major aortopulmonary collaterals. Ann Thorac Surg. 2013;95:1417-21.

4. Watanabe N, Mainwaring RD, Reddy VM, Palmon M, Hanley FL. Early complete repair of pulmonary atresia with ventricular septal defect and major aortopulmonary collaterals. Ann Thorac Surg. 2014;97:909-15.

5. Griselli M, McGuirk SP, Winslaw DS, Stumper O, de Giovanni JV, Miller P, et al. The influence of pulmonary artery morphology on the results of operations for major aortopulmonary collateral arteries and complex congenital heart defects. J Thorac Cardiovasc Surg. 2004;127:251-8.

6. Carotti A, Albanese SB, Filleppelli S, Rava L, Guccione P, Pongiglione G, et al. Determinants of outcome after surgical treatment of pulmonary atresia with ventricular septal defect and major aortopulmonary collateral arteries. J Thorac Cardiovasc Surg. 2010;140:1092-103.

7. Davies B, Mussa S, Davies P, Stickley J, Jones TJ, Barron DJ, et al. Unifocalization of major aortopulmonary collateral arteries in pulmonary atresia with ventricular septal defect is essential to achieve excellent outcomes irrespective of native pulmonary artery morphology. J Thorac Cardiovasc Surg. 2009;138: 1269-75.

8. Amark KM, Karamlou T, O'Carroll A, MacDonald C, Freedom RM, Yoo SJ, et al. Independent risk factors associated with mortality, reintervention, and achievement of complete repair in children with pulmonary atresia with ventricular septal defect. J Am Coll Cardiol. 2006;47:1448-56.

9. Song S, Park HK, Park YH, Cho BK. Pulmonary atresia with ventricular septal defects and major aortopulmonary collateral arteries. Circ J. 2009; 73:516-22.

10. Mainwaring RD, Patrick WL, Roth S, Kamra K, Wise-Faberowski L, Palmon M et al. Surgical algorithm and results for patients with pulmonary atresia with ventricular septal defect and major aortopulmonary collateral arteries. J Thorac Cardiovasc Surg. 2018;156:1194-204.

11. Mainwaring RD, Reddy VM, Peng L, Kuan C, Palmon M, Hanley FL. Hemodynamic assessment after complete repair of pulmonary atresia/major aortopulmonary collaterals. Ann Thorac Surg. 2013;95:1397-402.

12. Mainwaring RD, Patrick WL, Punn R, Palmon M, Reddy VM, Hanley FL. Fate of right ventricle to pulmonary artery conduits following complete repair of pulmonary atresia with major aortopulmonary collaterals. Ann Thorac Surg. 2015;99: 1685-91.

13. Mainwaring RD, Patrick WL, Ma M, Hanley FL. An analysis of patients requiring unifocalization revision following midline unifocalization for pulmonary atresia with ventricular septal defect and major aortopulmonary collaterals. Eur J Cardiothorac Surg. 2018;54:63-70.

14. Mainwaring RD, Hanley FL. Surgical techniques for repair of peripheral pulmonary artery stenosis. Semin Thorac Surg. 2017;29:198-205.

15. Mainwaring RD, Sheikh AY, Reddy VM, Punn R, Hanley FL. Surgical results in patients with pulmonary atresia/major aortopulmonary collaterals and Alagille syndrome. Eur J Cardiothorac Surg. 2012;42:235-41.

16. Brown SC, Eyskens B, Mertens L, Dumoulin M, Gewillig M. Percutaneous treatment of stenosed major aortopulmonary collaterals with balloon dilation and stenting: what can be accomplished? Heart. 1998;79:24-8.

17. Eldemire RS, Argueta-Morales IR, Gapsis B, Berman D, Hannan R, Nykanen D, et al. Assessment of vascular growth after surgical and transcatheter interventions in patients with pulmonary atresia with ventricular septal defect and major aortopulmonary collaterals. J Am Coll Surgeons. 2014;219(Suppl):S32.

18. Hoashi T, Yazaki S, Kagisaki K, Kitano M, Shimada M, Shiraishi I, et al. Importance of multidisciplinary management of pulmonary atresia with ventricular septal defect and major aortopulmonary collateral arteries. Gen Thorac Cardiovasc Surg. 2017;65:337-42.

19. d'Udekem Y, Alphonso N, Norgaard MA, Cochrane AD, Grigg LE, Wilkinson JL, et al. Pulmonary atresia with ventricular septal defect and major aortopulmonary collateral arteries; unifocalization brings no long-term benefits. J Thorac Cardiovasc Surg. 2005;130:1496-502.

20. Brizard CP, Liava'a M, d'Udekem Y. Pulmonary atresia, VSD and MAPCAs: results without unifocalization. Semin Thorac Cardiovasc Surg Pediatr Card Surg Апnu. 2009;12:139-44.

21. Liava'a M, Brizard CP, Konstantinov IE, Robertson T, Cheung MM Weintraub R, et al. Pulmonary atresia, ventricular septal defect, and major aortopulmonary collaterals: neonatal pulmonary artery rehabilitation without unifocalization. Ann Thorac Surg. 2012;93:185-92.

22. Soquet J, Liava'a M, Eastaugh L, Konstantinov IE, Brink J, Brizard CP, et al Achievements and limitations of a strategy of rehabilitation of native pulmonary 
vessels in pulmonary atresia with ventricular septal defect and major aortopulmonary collateral arteries. Ann Thorac Surg. 2017;103:1519-26.

23. Kim H, Sung SC, Choi KH, Lee HD, Ban GH, Chang YH. A central shunt to rehabilitate diminutive pulmonary arteries in patients with pulmonary atresia with ventricular septal defect. J Thorac Cardiovasc Surg. 2015;149: 515-20.

24. Mumtaz MA, Rosenthal G, Qureshi A, Prieto L, Preminger T, Lorber R, et al. Melbourne shunt promotes growth of diminutive central pulmonary arteries in patients with pulmonary atresia, ventricular septal defect, and systemic-topulmonary collateral arteries. Ann Thorac Surg. 2008;85:2079-83.

25. Hibino N, He D, Yuan F, Yu JH, Jonas R. Growth of diminutive central pulmonary arteries after right ventricle to pulmonary artery homograft implantation. Ann Thorac Surg. 2014;97:2129-33.

26. Chen Q, Ma K, Hua Z, Yang K, Zhang H, Wang X, et al. Multistage pulmonary artery rehabilitation in patients with pulmonary atresia, ventricular septal defect and hypoplastic pulmonary artery. Eur J Cardiothorac Surg. 2016;50:160-6.

27. Zhang Y, Hua Z, Yang K, Zhang H, Yan J, Wang X, et al. Outcomes of the rehabilitative procedure for patients with pulmonary atresia, ventricular septal defect and hypoplastic pulmonary arteries beyond the infant period. Eur J Cardiothorac Surg. 2014;46:297-303

28. Dragulescu A, Kammache I, Fouilloux V, Amedro P, Metras D, Kreitmann B, et al. Long-term results of pulmonary artery rehabilitation in patients with pulmonary atresia, ventricular septal defect, pulmonary artery hypoplasia, and major aortopulmonary collaterals. J Thorac Cardiovasc Surg. 2011;142:1374-80.

Key Words: congenital heart disease, pulmonary artery, pulmonary valve

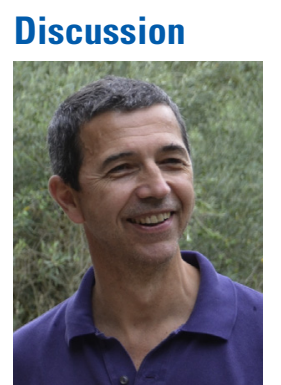

Dr Christian P. Brizard (Parkville, Australia). Thank you very much, Dr Mainwaring, for this nice presentation and for sending me all the data beforehand. Your team at Stanford has put a lot of effort into promoting and educating the community in the technique that you are advocating, and the goal has been attained many times over, and we want to congratulate you for that, too.

Your presentation today exposes the last aspect that has not been fully investigated: The need for revision of the unifocalized pulmonary vascular bed, which is a surrogate for the fate of the unifocalized pulmonary vascular bed. The article and presentation are clean and didactic; however, it seems that the weakness comes from the follow-up. This is very important because it changes the perspective of your presentation from a how-to-do-it to a broad investigation of the risk of needing a revision to the unifocalized vascular bed.

Your presentation and article assume that patients who do not have a reoperation at Stanford are well and have not yet required reoperation. You have told us that your series spanned 16 years and the median age at the time of unfocalization was 4 months with a median follow-up time of 4.7 years. We know that many of your patients are referred from outside your catchment area, to the credit of your team.
The first question is, can you confirm that in your mind the series presented today is a true representation of the risk for the need of unfocalization revision and what is the evidence that you have to support it?

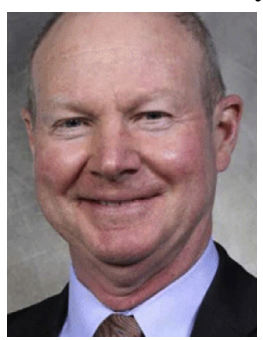

Dr Richard D. Mainwaring (Stanford, Calif). Thanks for your comment. It is correct that $90 \%$ of our patients come from outside our catchment area. These families come from hundreds, sometimes thousands, of miles away. The children have surgery, they convalesce in our unit, and then they go home. So it would be somewhat naive to think that is the end of the story.

Because these are complicated patients, we put together a surveillance program, the details of which are as follows: at 3 months we have an echocardiogram and oxygen saturations performed; at 6 months, those 2 studies plus a lung perfusion scan; at 9 months, another echocardiogram; and at 12 months, a cardiac catheterization is done. Essentially, if patients begin to show increases in right ventricle pressure, which many times the echocardiogram will indicate, then these investigations can be performed earlier.

An interesting thing is that it turns out that almost all of these patients you know about pretty early on. For example, the patients who developed increased pulmonary to aortic pressure show up pretty early. These patients underwent reoperations at a median time of 9 months. So essentially whatever happens to these reconstructed beds, the remodeling seems to occur fairly soon and is either stable or not so stable. I think for the same reason these patients are referred to us initially, they are going to be referred back if they need something done. I think they are very happy with the distal unifocalized bed. So I am very confident that we see the patients who need something done.

Fortunately, the group that needs something done is maybe 1 out of 5 patients, and 4 out of 5 patients go along, and of course the next intervention is going to be when the conduit fails. We know that a lot of conduits are done at outside institutions by surgeons who feel comfortable doing that. Through a combination of our surveillance program and then the reoperations that are sort of built into this program, we get quite a lot of information about the patients for whom we initially performed the unfocalization.

Dr Brizard. Thank you. My second question relates to the subgroup with unfocalization on shunt. Sixteen of 30 survivors required revision of their unfocalization. What would be the reason for the failure to achieve secondary suitability for repair? Would that be a lack of growthand coming from Melbourne I would have the tendency to think that it is the case-or would there be a secondary stenosis related to a high-pressure regimen or would it be technical issues? 
Dr Mainwaring. That's a good question. Clearly the patients who are not able to have a repair are different from the patients who are. They separate themselves away physiologically very early on. These are patients who have a lower oxygen saturation preoperatively and at cath they have a Qp:Qs $<1$. They tell you that they are different. When I go to the operating room, I walk in, the first thing I look at is the oxygen saturation monitor, and if it's $84 \%$ to $85 \%$ or higher, I know we are going to do a single-stage complete repair. If it's $75 \%$, then I know we will be doing a flow study. But those patients physiologically are different; their pulmonary vascular beds are less well developed. It's not a surprise then that the mortality in that group is 3.5 times higher, and it's not a surprise that the results of unfocalization revision are also not nearly as successful.

Dr Brizard. Thank you. My last questions are technical because the surgery presented today is truly spectacular. How do you recline the lung parenchyma circularly from the intermediate and lower branches? Do you cut through the lung parenchyma? Do you burn it? How do you deal with the air leaks? What do you with the pulmonary veins? Do you sometimes have to cut the upper pulmonary vein and then reconstruct it to get access to the intermediate pulmonary artery branches? Do you ever use simultaneous or sequential hybrid procedures? What advice do you give to teams who have no or little access to pulmonary homografts?

Dr Mainwaring. Thanks for the comments. First, the dissection is along the plane of the arteries and it goes to the edges of the parenchyma. Typically, we try to push the lung tissue away. We try not to get into the lung parenchyma, but if you do, those little air leaks will seal within hours, so that's really not a big problem. The pulmonary veins we never divide. There is a plane beneath the pulmonary vein where the reconstructed bed is, so we just sort of lift the pulmonary vein up and away.

Dr Brizard. But you go far beyond the upper pulmonary vein?

Dr Mainwaring. We go underneath the pulmonary vein and way beyond that on both sides. Regarding your question about homografts, we are fortunate to have access to a good supply. We fortunately have plenty of that. We believe that it is the ideal choice. In the absence of that, I would use anything that doesn't have glutaraldehyde, so fresh autologous pericardium. I don't know the specific answer.

Dr Brizard. Thank you.

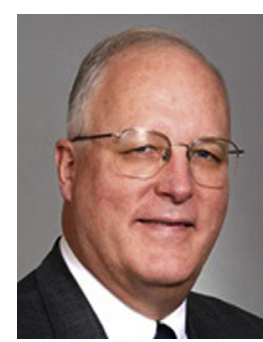

Dr John E. Mayer (Boston, Mass). I am surprised that there is not an interventional catheterization intervention center being reported here. My question is, what do you see as the role for those in dealing with these residual or peripheral pulmonary artery stenoses?

Dr Mainwaring. It's a spot-on question. Obviously, these are complementary things. In the illustration that I showed you with long segment stenoses, that's something that we would feel very strongly needs to be done surgically; in fact, most of the peripheral reconstructions we prefer to do surgically. Interventional cardiologists play an important and complementary role in our algorithm. Many of their interventions are directed more centrally. For example, they can dilate or stent the conduits and extend the longevity for an additional time period. Another example would be our experience with 15 or 16 patients who have undergone implantation of Melody valves (Medtronic, Minneapolis, Minn). Obviously, in these select patients, their peripheral unifocalized bed was found to be in good shape and so they could have a Melody valve and not have surgery.

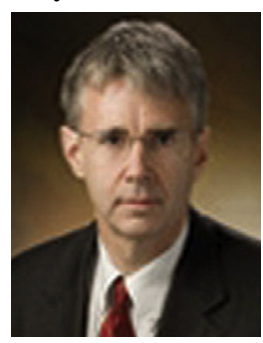

Dr J. William Gaynor (Philadelphia, $P a)$. I would like to ask 1 more question about that. In patients who are doing well that you are not following at your institution, do you know for sure that they haven't had an interventional cath and had dilations or even a stent placed? That's going to be necessary to know the true reintervention rate, and if they are not under your control, God knows what can happen.

Dr Mainwaring. Can I say absolutely that no one has had a stent? Probably not. I think the majority of the cardiologists, before they do any intervention, they will call us and ask us for advice.

There is also a large cohort of patients who achieved a good result from their unifocalization and were turned over to the referring groups permanently if there is no problem peripherally and do not need anything done to the peripheral bed. In this case, when the conduit fails and they need a conduit replacement, many groups are quite comfortable performing that surgery. We know of at least 25 patients who have had unifocalization performed at Stanford and subsequently had conduit replacements performed at other outside institutions. 

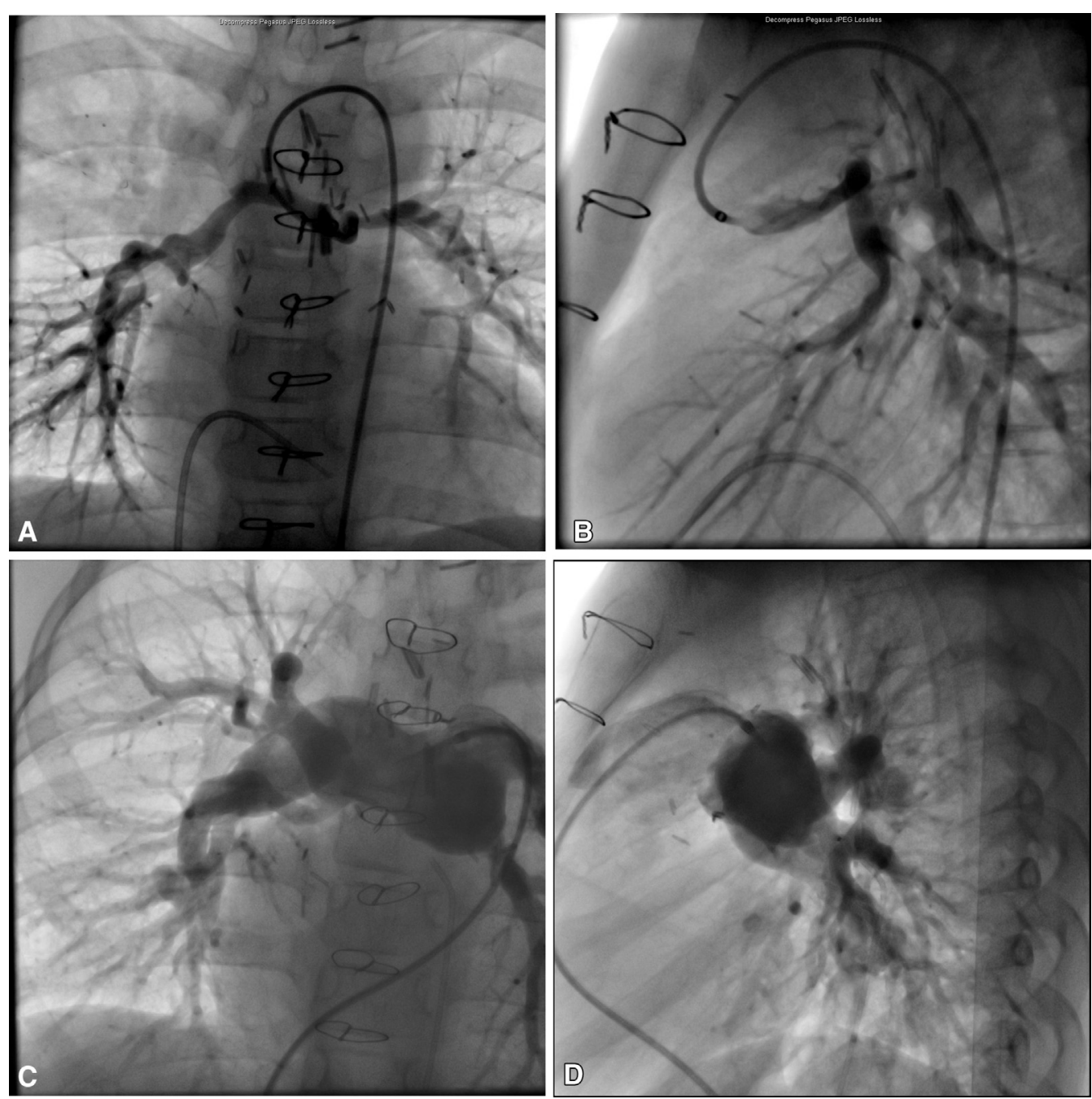

FIGURE E1. Angiograms of a patient who initially underwent a unifocalization and placement of a shunt. A and B, The anterior/posterior and lateral images demonstrating multiple, bilateral stenoses. This patient subsequently underwent unifocalization revision with concomitant intracardiac repair. C and $\mathrm{D}$, The follow-up catheterization images. 